\title{
103. Asymptotic Formulas with Sharp Remainder Estimates for Eigenvalues of Elliptic Operators of Second Order
}

\author{
By Hideo TAmura \\ Department of Applied Physics, Nagoya University \\ (Communicated by Kôsaku YosidA, M. J. A., Nov. 12, 1981)
}

1. Introduction. In this paper it is reported that the best possible remainder estimate is obtained in the asymptotic formula for eigenvalues of elliptic operators of second order on $\mathbf{R}_{x}^{n}$. Let $A\left(x, D_{x}\right)$ be a symmetric elliptic operator of the form

$$
A\left(x, D_{x}\right)=\sum_{j, k=1}^{n} D_{j} a_{j k}(x) D_{k}+V(x), \quad D_{j}=-i \partial / \partial x_{j} .
$$

We make the following assumptions on the coefficients. To describe the assumptions, we follow the standard multi-index notations and write $\langle x\rangle=\left(1+|x|^{2}\right)^{1 / 2}$.

Assumption (I). (a.1) $A\left(x, D_{x}\right)$ is uniformly elliptic ;

(a.2) $\left|\partial_{x}^{\alpha} a_{j k}(x)\right| \leqq C_{\alpha}\langle x\rangle^{-|\alpha|}$.

$$
\sum_{j, k=1}^{n} a_{j k}(x) \xi_{j} \xi_{k} \geqq \delta|\xi|^{2}, \quad \delta>0 .
$$

Assumption (II). There exists a constant $m, m>0$, such that: (V.1) $C_{1}\langle x\rangle^{m} \leqq V(x) \leqq C_{2}\langle x\rangle^{m}$; (V.2) $\left|\partial_{x}^{\alpha} V(x)\right| \leqq C_{\alpha}\langle x\rangle^{m-|\alpha|}$; (V.3) for $x,|x| \geqq R$ ( $R$ large enough),

$$
\sum_{j=1}^{n} x_{j}\left(\partial / \partial x_{j}\right) V(x) \geqq C_{3}\langle x\rangle^{m} .
$$

Under Assumptions (I) and (II), $A\left(x, D_{x}\right)$ admits a unique (positive) self-adjoint realization in $L^{2}\left(\mathbf{R}_{x}^{n}\right)$. We denote it by $A$. The operator $A$ has an infinite sequence of eigenvalues, $\left\{\lambda_{j}\right\}_{j=1}^{\infty}$, diverging to infinity. Let $N(\lambda), \lambda>0$, denote the number of eigenvalues less than $\lambda$ with repetition according to the multiplicities; $N(\lambda)=\sum_{\lambda_{j}<\lambda} 1$. One of the most important problems in the spectral theory is to derive the asymptotic formula with the best possible remainder estimate for $N(\lambda)$ as $\lambda \rightarrow \infty$.

Now, we shall state the result.

Theorem 1. Assume that $n \geqq 2$ and that Assumptions (I) and (II) are satisfied. Let

$$
A_{0}(x, \xi)=\sum_{j, k=1}^{n} a_{j k}(x) \xi_{j} \xi_{k}+V(x)
$$

Then,

$$
N(\lambda)=(2 \pi)^{-n} \iint_{A_{0}(x, \xi)<\lambda} d \xi d x\left(1+O\left(\lambda^{-(1 / 2+1 / m)}\right)\right)
$$

as $\lambda \rightarrow \infty$. 
Remark. Under the assumptions above, the remainder estimate is the best possible one, which is seen from the example of harmonic oscillation with $V(x)=|x|^{2}$ and $\alpha_{j k}(x)=\delta_{j k}, \delta_{j k}$ being Kronecker's delta.

Recently, Helffer and Robert [2] have obtained the best possible remainder estimate, including the case $n=1$, when $A_{0}(x, \xi)$ is quasihomogeneous in the sense that $A_{0}\left(r^{g} x, r^{d} \xi\right)=r^{M} A_{0}(x, \xi), r>0$, for some integers $g, d$ and $M(\geqq 1)$. In our situation, this implies that $a_{j k}(x)$ $=a_{j k}^{(0)}$ (constant) and $V(x)$ is a homogeneous polynomial. Another recent results related to Theorem 1 can be found in [1], [3], [5], [6] and [7].

2. Sketch of proof. For the proof, we shall give only a sketch, restricting ourselves to the Schrödinger operator $H=-\Delta+V$ with $V(x)$ satisfying (V.1)-(V.3). The detailed proof will be published elsewhere ([8]).

The idea of proof is basically due to Hörmander [4]. However, we work not only in $L^{2}\left(\mathbf{R}_{x}^{n}\right)$ but also in the space $L^{2}\left(\mathbf{R}_{\xi}^{n}\right)=\{(\mathcal{F} f)(\xi): f$ $\left.\in L^{2}\left(\mathbf{R}_{x}^{n}\right)\right\}$, where $\mathcal{F}$ denotes the Fourier transform. Also, we denote by $\mathcal{F}^{*}$ the inverse Fourier transform.

We denote by $\left\{u_{j}\right\}_{j=1}^{\infty}$ a system of the normalized eigenfunctions of $H$ corresponding to the eigenvalues $\left\{\lambda_{j}\right\}_{j=1}^{\infty}$. Let $H_{0}(x, \xi)=|\xi|^{2}+V(x)$. For $\lambda$ large enough, we decompose the phase space into the following three regions :

$$
\begin{aligned}
& \Omega_{1}(\lambda)=\left\{(x, \xi): \lambda / 2<H_{0}<2 \lambda,|\xi|>|x|^{m / 2}\right\}, \\
& \Omega_{2}(\lambda)=\left\{(x, \xi): \lambda / 2<H_{0}<2 \lambda,|x|>|\xi|^{2 / m}\right\}, \\
& \Omega_{3}(\lambda)=\left\{(x, \xi): H_{0}<\lambda / 2 \text { or } H_{0}>2 \lambda\right\} .
\end{aligned}
$$

(The decomposition made actually in the proof is a little more complicated.) Let $\omega_{k}(x, \xi ; \lambda), 1 \leqq k \leqq 3$, be a real symbol with support in $\Omega_{k}(\lambda)$ and define $n_{k}(\lambda)$ by

$$
n_{k}(\lambda)=\sum_{\lambda_{j}<\lambda}\left\|\omega_{k}\left(x, D_{x} ; \lambda\right) u_{j}\right\|^{2},
$$

where \|\| denotes the $L^{2}$ norm in $L^{2}\left(\mathbf{R}_{x}^{n}\right)$. We derive the asymptotic formula for each $n_{k}(\lambda)$ as $\lambda \rightarrow \infty$.

Roughly speaking, the asymptotic formula for $n_{1}(\lambda)$ is derived by constructing the approximate expression (parametrix) for $\omega_{1}\left(x, D_{x} ; \lambda\right)^{*} \exp (-i t H) \omega_{1}\left(x, D_{x} ; \lambda\right)$. The construction is based on the theory of Fourier integral operators. Here it should be noted that we do not take any fractional power of $H$.

On the other hand, we work in $L^{2}\left(\mathbf{R}_{\xi}^{n}\right)$ to derive the asymptotic formula for $n_{2}(\lambda)$. In this space, the operator $H$ is transformed into $\mathscr{H}^{*}=\mathcal{F}_{H} \mathcal{F}^{*}$. We apply to $\mathscr{H}$ the same argument as in the derivation of the asymptotic formula for $n_{1}(\lambda)$.

The asymptotic formula for $n_{3}(\lambda)$ is rather easy to derive, because the hypersurface (energy level) $E_{0}(\lambda)=\left\{(x, \xi) ; H_{0}(x, \xi)=\lambda\right\}$ is cut off by 
the symbol $\omega_{3}(x, \xi ; \lambda)$. Indeed, this is done by using the theory of pseudo-differential operators only.

After establishing the asymptotic formula for each $n_{k}(\lambda)$, Theorem 1 is proved by use of a partition of unity in the phase space.

3. Asymptotic formula for bound states. The method stated above is useful in deriving the asymptotic formula for bound states (negative eigenvalues) of Schrödinger operators.

Let $H=-\Delta-V$ be the Schrödinger operator acting on $L^{2}\left(\mathbf{R}_{x}^{n}\right)$. For the potential $V(x)$, we assume that:

(A.1) $V(x)$ is decomposed as $V(x)=V_{1}(x)+V_{2}(x)$;

(A.2) $\quad V_{1}(x)^{-1}$ satisfies Assumption (II) with $m, 0<m<2$;

(A.3) $\quad V_{2}(x)$ is real-valued and belongs to $L^{n / 2}\left(\mathbf{R}_{x}^{n}\right)$.

If $n \geqq 3$ and if $V(x)$ satisfies Assumptions (A.1)-(A.3), then $H$ admits a unique self-adjoint realization (Friedriches' extension) in $L^{2}\left(\mathbf{R}_{x}^{n}\right)$. We denote it by $H$ also. Furthermore, $H$ has an infinite sequence of negative eigenvalues, $\left\{\lambda_{j}\right\}_{j=1}^{\infty}$, approaching zero. Let $N(\lambda), \lambda>0$, be the number of eigenvalues less than $-\lambda$ with repetition according to the multiplicities; $N(\lambda)=\sum_{\lambda_{j}<-\lambda} 1$. We obtain the following asymptotic formula for $N(\lambda)$ as $\lambda \rightarrow 0$.

Theorem 2. Assume that $n \geqq 3$ and that Assumption (A.1)-(A.3) are satisfied. Then,

$$
N(\lambda)=(2 \pi)^{-n} \iint_{|\xi|^{2}-V(x)<-\lambda} d \xi d x\left(1+O\left(\lambda^{1 / m-1 / 2}\right)\right)
$$

as $\lambda \rightarrow 0$.

Remark. Theorem 2 covers the case of the Coulomb potential $1 /|x|$ and this example shows that the remainder estimate above is the best possible one.

\section{References}

[1] V. Guillemin and S. Sternberg: Some problems in integral geometry and some related problems in micro-local analysis. Amer. J. Math., 101, 915955 (1979).

[2] B. Helffer and D. Robert: Comportement semi-classique du spectre des hamiltoniens quantiques hypoelliptiques. C. R. Acad. Sci. Paris, serié I, 292, 47-50 (1981).

[3] - : Comportement asymptotique précisé du spectre d'opérateurs globalement elliptiques dans $R^{n}$. ibid., serié I, 292, 363-366 (1981).

[4] L. Hörmander: The spectral function of an elliptic operator. Acta. Math., 121, 193-218 (1968).

[5] - : On the asymptotic distribution of the eigenvalues of pseudodifferential operators in $R^{n}$. Ark. Mat., 17, 297-313 (1979).

[6] D. Robert: Propriétés spectrales d'opérateurs pseudo-différentiels. Comm. in P. D. E., 3, 755-826 (1978).

[7] M. A. Shubin and V. N. Tulovskii: On the asymptotic distribution of the 
eigenvalues of pseudo-differential operators in $R^{n}$. Mat. Sb., 92, 565-583 (1973).

[8] H. Tamura: Asymptotic formulas with sharp remainder estimates for eigenvalues of elliptic operators of second order (preprint).

[9] — : Asymptotic formula with sharp remainder estimates for bound states of Schrödinger operators, I and II (preprints). 